ARTICLE

\title{
Room-temperature single-photon source with near-millisecond built-in memory
}

\author{
Karsten B. Dideriksen (10 ${ }^{1}$, Rebecca Schmieg (1) ${ }^{1}$, Michael Zugenmaier (iD ${ }^{1}$ \& Eugene S. Polzik (i) ${ }^{1 凶}$
}

Non-classical photon sources are a crucial resource for distributed quantum networks. Photons generated from matter systems with memory capability are particularly promising, as they can be integrated into a network where each source is used on-demand. Among all kinds of solid state and atomic quantum memories, room-temperature atomic vapours are especially attractive due to their robustness and potential scalability. To-date room-temperature photon sources have been limited either in their memory time or the purity of the photonic state. Here we demonstrate a single-photon source based on room-temperature memory. Following heralded loading of the memory, a single photon is retrieved from it after a variable storage time. The single-photon character of the retrieved field is validated by the strong suppression of the two-photon component with antibunching as low as $g_{\mathrm{RR} \mid \mathrm{W}=1}^{(2)}=0.20 \pm 0.07$. Non-classical correlations between the heralding and the retrieved photons are maintained for up to $\tau_{\mathrm{NC}}^{\mathcal{R}}=(0.68 \pm 0.08) \mathrm{ms}$, more than two orders of magnitude longer than previously demonstrated with other room-temperature systems. Correlations sufficient for violating Bell inequalities exist for up to $\tau_{\mathrm{BI}}=(0.15 \pm 0.03) \mathrm{ms}$.

\footnotetext{
${ }^{1}$ Niels Bohr Institute, University of Copenhagen, Copenhagen $\varnothing$, Denmark. ${ }^{凶}$ email: polzik@nbi.ku.dk
} 
$\mathrm{R}$ emarkable progress has been achieved with deterministic solid-state single-photon sources ${ }^{1-7}$. However, these sources require cryogenic temperatures to allow efficient photon interference ${ }^{1,2,8}$. Ultracold atoms, which has been another system of choice ${ }^{9-13}$, offer excellent performance at the expense of complexity of the experimental apparatus.

In comparison, room-temperature atomic systems have attracted a lot of attention due to their potential scalability, robustness, natural compatibility with atomic memories and favorable duty cycle $^{14-18}$. The pioneering DLCZ proposal ${ }^{19}$ provided the route towards using an ensemble of atoms to combine single-photon generation and storage in the same system offering experimental simplicity, while enabling quantum information processing ${ }^{20}$ and quantum communication ${ }^{21}$ schemes. Envisioning quantum networks on a continental scale calls for single-photon sources capable of generating a photon on demand within a storage time comparable to photon time-offlight between the parties, i.e., in the millisecond regime. When it comes to quantum storage time, the main challenge with roomtemperature atomic ensembles is their thermal motion and/or collisional decoherence. Due to those limitations the DLCZ-type single-photon sources in room-temperature vapors have been up to now limited in their on-demand retrieval time to a few microseconds ${ }^{22-24}$. Another limitation on the performance of DLCZ-type sources has been the quantum readout noise ${ }^{25}$. An alternative route to a single-photon source on demand is to generate a single photon in one system, store it in another memory system and then retrieve it on demand. Towards realization of this approach in room-temperature gasses, storage of weak classical pulses limited to a few microseconds $26-28$ and reaching a few milliseconds ${ }^{29}$ has been demonstrated. Although the storage of external classical light pulses on the time scale of a second ${ }^{30}$ has recently been reported, its applicability to singlephoton communication remains to be demonstrated.

Here we demonstrate an ensemble-based, deterministic roomtemperature single-photon source exhibiting clear antibunching and a non-classical memory of $0.68(8) \mathrm{ms}$, two orders of magnitude longer than previously demonstrated with other roomtemperature system $\mathrm{s}^{22,23}$. This has been achieved by combining three main ingredients, the principle of motional averaging 25,31 , a spin-protecting coating on the walls of the atomic vapor cell ${ }^{32}$, and the use of a Raman transition at the "magic detuning" for writing and retrieving the single photon.

\section{Results}

Experimental principle. We create a single collective excitation of the atomic ensemble when a heralding "write" photon generated via spontaneous Raman scattering is detected (Fig. 1a). Usually, the Gaussian transverse profile of the excitation beam leads to in-homogeneous coupling to the atoms, and therefore the detection of the heralding photon corresponds only to a snapshot of the atomic positions. Consecutive atomic motion changes these positions and renders the subsequent retrieval of the photon inefficient. To remedy the effect of atomic motion, we use motional averaging to project the ensemble onto the symmetric Dicke state with equal weights for all atoms ${ }^{33}$. This is achieved by narrowband filter cavities (Fig. 1c), extending the duration of the detection mode of the heralding photon beyond the transverse transit time of atoms through the cell channel. As the atoms travel through the beam, the random delay from the filter cavity leads via motional averaging to washing out the which-path information of the photon, and thus equalizing the contribution of all atoms to the single collective excitation. The antirelaxation coating of the walls preserves the spin state of the atoms for thousands of collisions, extending the lifetime of the symmetric

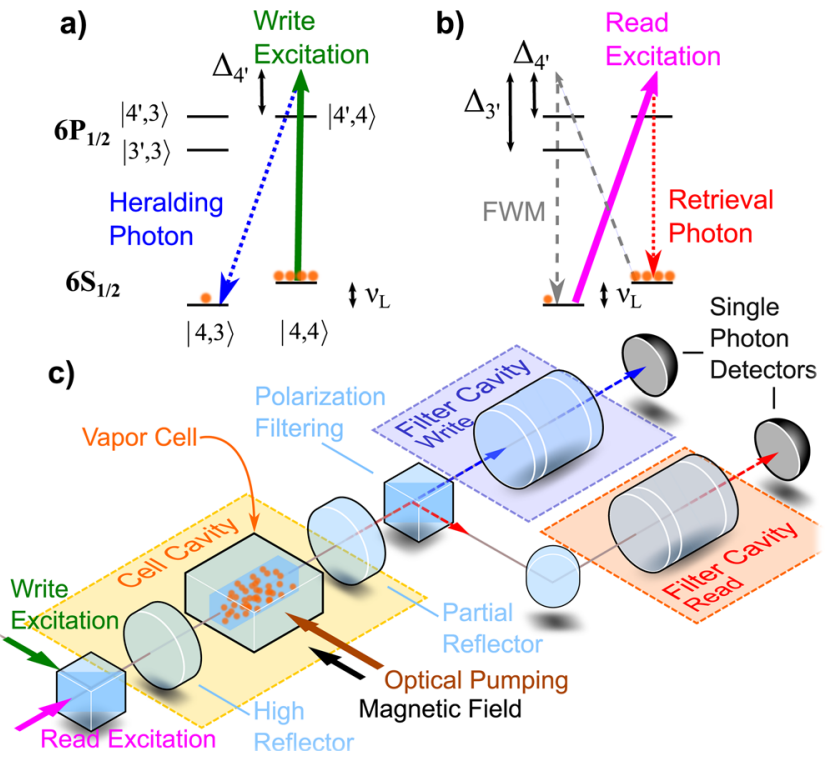

Fig. 1 Experimental setup and excitation schemes. a Write excitation scheme: $\pi$-polarized, far-detuned excitation light creates an atomic excitation via a Raman scattering process. Only relevant atomic levels shown. b Read excitation scheme: $\sigma$-polarized light used to retrieve stored excitation via Raman scattering and scattering desired deterministic single photon. Excess four-wave-mixing (FWM) noise is suppressed by choosing $\Delta_{4^{\prime}}=924 \mathrm{MHz}$. c Schematic of simplified experimental setup including paths for write and read scattered photons through polarization and spectral filtering.

collective excitation. Four-wave-mixing (FWM) noise has been identified to be the main limitation for room-temperature vapor schemes ${ }^{25,34,35}$. Several strategies have been pursued to suppress this noise including ladder schemes ${ }^{17,18}$, cavity engineering ${ }^{36}$, absorption $^{37}$, or Raman absorption ${ }^{38}$. An idea that is also suitable for Raman transitions between Zeeman levels is to use polarization selection rules ${ }^{35,39}$. However, as a result of interfering excitation paths, this generally suppresses the Raman transitions as shown in ref. ${ }^{40}$. Here, we turn this effect to our advantage, exploiting a magic detuning (Fig. 1b) to suppress only the undesired FWM transition by the destructive interference of Raman amplitudes via coupling to different excited states (see "Methods" section).

Experimental setup. In the experiment the atomic ensemble is a thermal cesium vapor with a cross-section of $300 \mu \mathrm{m} \times 300 \mu \mathrm{m}$ and a length of $10 \mathrm{~mm}$. The small cell cross-section enables fast motional averaging and high intensity of the laser pulses. The $N$ atoms of the atomic ensemble are initially optically pumped into the coherent spin state $|g\rangle=\left|g_{1} \ldots g_{\mathrm{N}}\right\rangle$, where $\left|g_{\mathrm{i}}\right\rangle=\left|F=4, m_{\mathrm{F}}=4\right\rangle$. Typically, we achieve an atomic polarization of $99.2 \%$. Afterwards, a collective excitation is written into the atomic ensemble with low probability using a fardetuned, $\pi$-polarized write pulse (Fig. 1a). Upon detection of a scattered heralding single photon, the atomic ensemble is ideally projected onto a long-lived, symmetric Dicke state $|s\rangle=$ $\sum_{i=1}^{N} \frac{1}{\sqrt{N}}\left|g_{1} \ldots s_{\mathrm{i}} \ldots g_{\mathrm{N}}\right\rangle$ acting as the memory storage state with $\left|s_{\mathrm{i}}\right\rangle=|4,3\rangle$. This choice of Zeeman configuration is beneficial for long storage time $\mathrm{e}^{30}$. The cell is subject to a magnetic field providing a frequency splitting of $v_{\mathrm{L}}=2.4 \mathrm{MHz}$ between the relevant Zeeman levels. To enhance light-atom interaction, the vapor cell is placed in an asymmetric linear cavity (Fig. 1c) with finesse $\mathcal{F} \approx 13$, a compromise between interaction enhancement and 
photon output coupling. The orthogonal polarization of the heralding photon with respect to the excitation light, and the relative detuning by one Larmor frequency $v_{\mathrm{L}}$ facilitates filtering of the heralding photon from the $10^{7}$ excitation photons in the same spatial mode by polarization filtering optics, and subsequent spectral filtering with narrowband filter cavities (Fig. 1c). The filter cavities simultaneously serve the purpose of motional averaging by adding random delays to the scattered photons ${ }^{31}$, selecting the symmetric Dicke state. Combining motional averaging and narrow-band filtering enables a heralding probability of $82 \%$ in the symmetric mode (see Supplementary Note 2).

After a variable delay $\tau_{\mathrm{D}}$, a $\sigma$-polarized read pulse retrieves the stored collective excitation coherently in form of a deterministic "retrieval" single photon (Fig. 1b). The filtered heralding and retrieval single photons are detected using two superconducting nanowire single-photon detectors (SNSPD).

The excitation light and single photons propagate along the cell axis orthogonal to the quantization axis defined by the optical pumping and magnetic field (Fig. 1c). The write excitation light in $\pi$-polarization (solid green arrow in Fig. 1a) generates the write photon (dashed blue) in the polarization mode orthogonal to the quantization axis. In the read process the latter polarization mode is used by the linearly-polarized excitation light. Beside the desired $\sigma_{+}$-polarized component (solid pink arrow in Fig. $1 \mathrm{~b}$ ) this linearly-polarized mode contains also the undesired $\sigma_{-}$-polarized component (dashed diagonal line in Fig. 1b), which drives the FWM process contaminating the stored excitation and adds noise to the retrieved light. By choosing the magic detuning $\Delta_{4^{\prime}}=924$ $\mathrm{MHz}$ for the read we effectively suppress this FWM process that turns out to be critical for the purity of the generated state.

The experimental sequence consists of two main parts, an optical pumping and locking window for all cavities, and a window containing the experimental write-read pulse sequence. The latter (Fig. 2a) contains a $350 \mu$ s optical pumping pulse for state initialization, a $40 \mu$ s write pulse, a variable delay $\tau_{\mathrm{D}}$, and a $200 \mu \mathrm{s}$ read pulse. All pulses are turned on and off smoothly to prevent high frequency harmonics which can falsely excite the memory state. The sequence of write, read and optical pumping pulses is repeated up to 75 times, depending on $\tau_{\mathrm{D}}$ before re-locking of the cavities becomes necessary. For delay times of $100 \mu$ s and longer, an additional repump pulse is used to counteract birefringence effects in the cell cavity due to the atomic polarization decay.

The retrieved light follows an exponentially decaying temporal envelope (Fig. 2b). One component of the atomic noise follows the same envelope while the second component grows linearly during the delay and the pulse duration (the noise origin is discussed below). Hence, the signal-to-noise ratio (SNR) depends on the time window chosen in post-processing. We find that truncating the write window to $31 \mu$ s out of the $40 \mu$ s pulse duration and the read window at $\tau_{\mathrm{R}}=40 \mu$ s offers a good tradeoff between SNR and retrieval efficiency, see Supplementary Note 4.

The temporal shape of the read noise validates that FWM noise is suppressed below other sources of read noise. Indeed, dominating FWM noise would mean that the read noise grows during the readout ${ }^{41}$, as was observed in ref. ${ }^{25}$. The exponential decay in the read noise shows that the FWM noise is negligible.

Photon correlations. The conditional generation of a single excitation in the atomic memory is characterized by the nonclassical cross-correlations between the single photons scattered during the write and consecutive read pulses. The relevant $2 \mathrm{nd}-$ order cross-correlation is given by $g_{\mathrm{WR}}^{(2)}=\left\langle n_{\mathrm{W}} n_{\mathrm{R}}\right\rangle /\left(\left\langle n_{\mathrm{W}}\right\rangle\left\langle n_{\mathrm{R}}\right\rangle\right)$ where $n_{\mathrm{W}}\left(n_{\mathrm{R}}\right)$ is the number of detection events during the write (read) process.

A long temporal shape of the retrieved light in the tens of microseconds range provides an advantage for the characterization

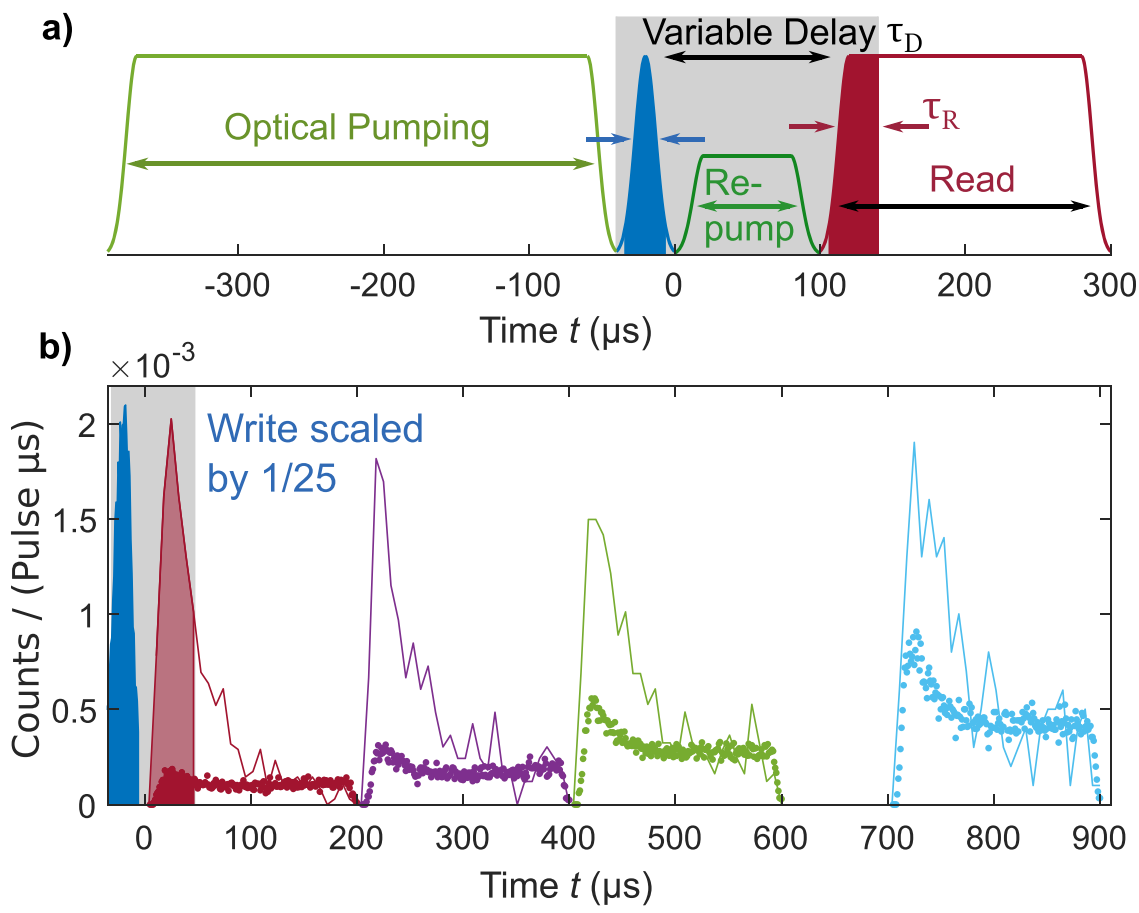

Fig. 2 Experimental pulse sequence and temporal shape of detection events. a Illustration of smoothened write, read and optical pumping pulses, variable delay between write and read, and optional repump for delayed readout. The solid areas represent integration windows used in the analysis. $\mathbf{b}$ Blue areadetected counts during heralding write pulses ( $31 \mu \mathrm{s}$, scaled 1/25). Solid curves-detected counts during read pulses ( $200 \mu \mathrm{s}$, delayed 10 to $710 \mu \mathrm{s}$ ) conditioned on the heralding write count (averaged over $7 \mu$ s bins). Dotted curves-the read noise level in the absence of write pulse ( $1 \mu$ s binning). Source data are provided as a Source Data file. 
of the photon source. Under those conditions the SNSPD, for which the dead time is less than $50 \mathrm{~ns}$, works as a photon-numberresolving detector. This capability allows for accurate accounting of multiphoton events, which would otherwise compromise the accuracy of the measurement of correlation functions.

In the absence of losses and extra noise the joint state of the write photon and the memory is of the two-mode squeezer type:

$$
\left|\Psi_{\text {uncond. }}\right\rangle=\sqrt{1-p_{0}}\left(|0\rangle_{\mathrm{W}}|0\rangle_{\mathrm{A}}+\sqrt{p_{0}}|1\rangle_{\mathrm{W}}|1\rangle_{\mathrm{A}}+p_{0}|2\rangle_{\mathrm{W}}|2\rangle_{\mathrm{A}}+\mathcal{O}\left(p_{0}^{3 / 2}\right)\right),
$$

where $p_{0}$ is the probability of creating one or more excited pairs. $|n\rangle_{\mathrm{W}}\left(|n\rangle_{\mathrm{A}}\right)$ refers to $n$ excitations of the write scattered field (symmetric excitations in the atomic ensemble). Thus, the multiple-pair excitation probability $p_{0}$ has to be kept low enough to avoid falsely heralding the single-pair state due to limited detection of the heralding field (e.g., propagation losses). We can directly relate the excitation probability to the mean number of excitations $\left\langle n_{\text {exc }}\right\rangle$ via $p_{0}=\left\langle n_{\text {exc }}\right\rangle /\left(1+\left\langle n_{\text {exc }}\right\rangle\right)$. For low number of excitations and neglecting noise, this gives $p_{0} \approx\left\langle n_{\mathrm{W}}\right\rangle / \eta_{\mathrm{X}}$, which is the mean number of detected write counts $\left\langle n_{\mathrm{W}}\right\rangle$ scaled with the write detection efficiency $\eta_{\mathrm{X}}=2.9 \%$ that includes the outcoupling from the cell cavity, propagation efficiencies through the filter setup and the quantum efficiency of the detector, see Supplementary Note 3.

With the decreased write pulse energy and thus $\left\langle n_{\mathrm{W}}\right\rangle, g_{\mathrm{WR}}^{(2)}$ grows as seen in Fig. $3 \mathrm{a}$ as a low multipair generation probability is crucial for a high cross-correlation between the write and read fields ${ }^{21}$. When $n_{\mathrm{W}}$ would be decreased even further, we expect the detection events of the write field to be dominated by background noise limiting the correlations. The high value of $g_{\mathrm{WR}}^{(2)} \approx 10$ obtained for low $n_{\mathrm{W}}$ testifies to the high heralding efficiency of the excitation storage in the memory and its consecutive readout.
The retrieval efficiency $\eta_{\mathrm{R}}=\left\langle n_{\mathrm{R} \mid \mathrm{W}=1}\right\rangle-\left\langle n_{\text {noise }}\right\rangle$ is defined as the difference between the mean number of counts conditioned on a single heralding write count, and the mean number of detected noise read counts in the absence of a write pulse (see Supplementary Note 4). As $\left\langle n_{\mathrm{W}}\right\rangle$ grows (Fig. $3 \mathrm{~b}$ ), $\eta_{\mathrm{R}}$ first grows rapidly as the write dark counts become negligible, and then continues to grow slower as the heralded state acquires an increasing contribution of multiple stored excitations.

To gain more insight, we model the system as a two-mode squeezed state with uncorrelated noise using probability generating functions (see Supplementary Note 3 ). The model yields the mean detected count rates, the cross-correlation, the retrieval efficiency and the conditional auto-correlation. The atomic noise contributions used in the model are found experimentally from the spectral scans of the filter cavities with and without sending a write pulse (see Supplementary Note 2). The only free fit parameters remaining in the model are the detection and intrinsic retrieval efficiencies. These are determined by simultaneously fitting to $g_{\mathrm{WR}}^{(2)}, \eta_{\mathrm{R}}$, and $\left\langle n_{\mathrm{R}}\right\rangle$. We observe good agreement of the experimental data with the fitted model as seen from Fig. 3. From the fit parameter we estimate the intrinsic retrieval efficiency, i.e., the efficiency of retrieving one excitation from the symmetric atomic mode into the cell cavity mode, to be $\eta_{\mathrm{R}}^{*}=(70 \pm 8) \%$ for $\tau_{\mathrm{R}}=40 \mu$ s (see "Methods" section). We note that the propagation losses through the filters are not due to a spectral mismatch of the retrieved photon.

Next, we demonstrate that the memory indeed stores a single excitation which can be deterministically retrieved on-demand as a single photon. Towards this end, we measure the conditional autocorrelation function and verify the sub-Poissonian character of the retrieved field, for which $g_{\mathrm{RR} \mid \mathrm{W}=1}^{(2)}<1$. Figure $3 c$ shows $g_{\mathrm{RR} \mid \mathrm{W}=1}^{(2)}$ as a function of $\left\langle n_{\mathrm{W}}\right\rangle$ which in the present case of number-resolving detection is defined as $g_{\mathrm{RR} \mid \mathrm{W}=1}^{(2)}=\left\langle n_{\mathrm{R} \mid \mathrm{W}=1}\left(n_{\mathrm{R} \mid \mathrm{W}=1}-1\right)\right\rangle /$
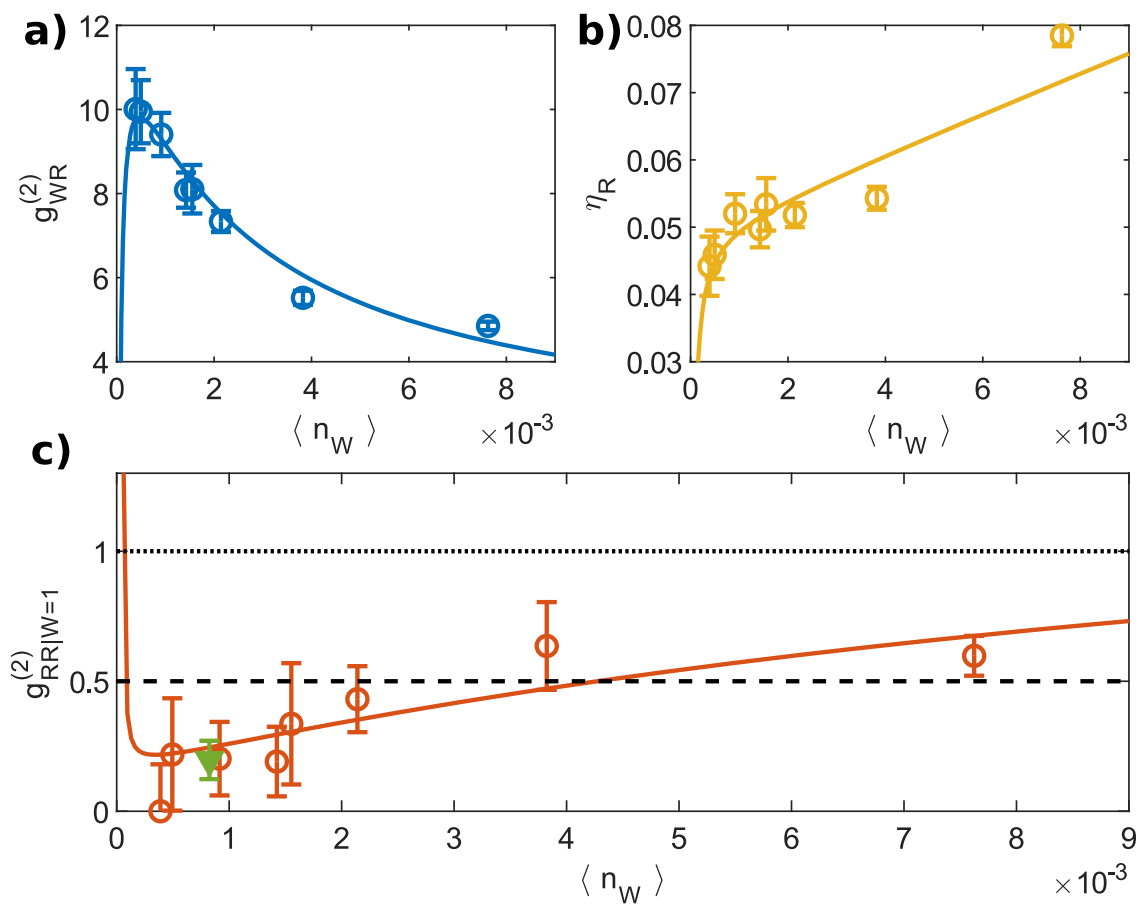

Fig. 3 Photon correlations and retrieval efficiency. a 2 nd-order cross-correlation function $g_{W R^{\prime}}^{(2)} \mathbf{b}$ retrieval efficiency $\eta_{\mathrm{R}}$ and $\mathbf{c} 2$ nd-order conditional autocorrelation function $g_{\mathrm{RR} \mid \mathrm{W}=1}^{(2)}$ of the retrieved light field. $g_{\mathrm{RR} \mid \mathrm{W}=1}^{(2)}=1$ (dotted line) is the classical limit and $g_{\mathrm{RR} \mid \mathrm{W}=1}^{(2)}=0.5$ (dashed line) is the two-photon Fock state auto-correlation value. All functions are plotted against the mean number of detected write counts $\left\langle n_{\mathrm{W}}\right\rangle$. Shown are measured data (circles) and the model (full lines). To improve the experimental uncertainty on $g_{\mathrm{RR} \mid \mathrm{W}=1}^{(2)}$ we combine points for $\left\langle n_{\mathrm{W}}\right\rangle<2 \times 10^{-3}$ (green triangle). Error bars represent one standard deviation. Source data are provided as a Source Data file. 
$\left\langle n_{\mathrm{R} \mid \mathrm{W}=1}\right\rangle^{2}$, where $n_{\mathrm{R} \mid \mathrm{W}=1}$ is the number of read detection events in each sequence with a preceding heralding write detection event. We observe good agreement between the experimental data and the model.

To improve the precision of $g_{\mathrm{RR} \mid \mathrm{W}=1}^{(2)}$ we combine datasets for $\left\langle n_{\mathrm{W}}\right\rangle<2 \times 10^{-3}$. According to the model, the read field is found to weakly depend on $p_{0}$ in this range. Under those conditions, the write-read sequence has been repeated $3 \times 10^{7}$ times. The observed heralded write probability of $\left\langle n_{\mathrm{W}}\right\rangle \sim 10^{-3}$ (green point in Fig. 3c) is comprised of $p_{0} \sim 0.03$ of the intrinsic write scattering probability and the write propagation efficiency of $\eta_{X}=0.029$. The overall write-read efficiency is $\eta_{\mathrm{tot}}=p_{0} \eta_{\mathrm{X}} \eta_{\mathrm{Y}} \sim 5 \times 10^{-5}$, where $\eta_{\mathrm{Y}}=0.06$ includes the intrinsic retrieval efficiency of 0.7 and the read propagation efficiency of 0.086 (see "Methods" section). The probability of the write event followed by the double retrieval event from the memory signifying the deviation from an ideal single photon storage and retrieval is $\sim 2 \times 10^{-7}$. The resulting $g_{\mathrm{RR} \mid \mathrm{W}=1}^{(2)}=0.20 \pm 0.07$ is a clear indication of the single photon nature of our source. Furthermore, there is an appreciable margin of more than four standard deviations to the two-photon Fock state auto-correlation $g_{\mathrm{n}=2, \mathrm{n}=2}^{(2)}=0.5$, which indicates good fidelity of the single-photon state.

Delayed readout and memory time. The quantum memory capabilities of the system are mapped out by varying the delay time between write and read pulses from 10 to $1010 \mu$ s. In Fig. 2b we have included the histograms for conditional read counts and unconditional read noise for various delays. While kept in the dark, the atoms decay into the storage state primarily due to wall collisions. This leads to an increase of the read noise with increasing delay times $\tau_{\mathrm{D}}$. Atomic decay compromises the readout in two ways, as atoms incoherently transferred into $|4,3\rangle$ contribute to the readout either by coupling to the easily retrievable symmetric mode or by coupling to the weakly retrieved asymmetric modes of the ensemble: 1 ) The high readout rate at the beginning of the read pulse originates from the symmetric mode which is efficiently read out. The retrieval of the incoherent excitation follows the same temporal shape as the desired stored excitation (Fig. 2b). These incoherent contributions to the symmetric mode constitute approximately half of the noise at the beginning of the read pulse (Fig. $2 \mathrm{~b}$ and Supplementary Note 2). 2) Incoherent contributions to the asymmetric modes are read out only inefficiently. The accumulation of incoherent population in $|4,3\rangle$ and hence asymmetric modes leads to a count rate that slowly increases over time as the population in $|4,3\rangle$ grows.

Both noise contributions grow approximately linearly with $\tau_{\mathrm{D}}$ degrading the SNR between conditional and unconditional readout.

Important characteristics of the on-demand single photon source enabled by the quantum memory are the 2nd-order crosscorrelation function $g_{\mathrm{WR}}^{(2)}$ and the Cauchy-Schwarz parameter $\mathcal{R}=\left(g_{\mathrm{WR}}^{(2)}\right)^{2} /\left(g_{\mathrm{RR}}^{(2)} g_{\mathrm{WW}}^{(2)}\right)$ versus delay time $\tau_{\mathrm{D}}{ }^{42}$. The latter can be used to quantify the non-classicality of correlations between write and read intensities. In Fig. 4 the respective values for $g_{\mathrm{WR}}^{(2)}\left(\tau_{\mathrm{D}}\right)$ and $\mathcal{R}\left(\tau_{\mathrm{D}}\right)$ are shown, along with the exponential fit following $g_{\mathrm{WR}}^{(2)}\left(\tau_{\mathrm{D}}\right)=B \cdot \exp \left(-\tau_{\mathrm{D}} / \tau_{\mathrm{M}}\right)+1 \cdot g_{\mathrm{WW}}^{(2)}$ is independent of $\tau_{\mathrm{D}}$ and because noise dominates the unconditional readout, the dependency for $g_{\mathrm{RR}}^{(2)}$ is marginal which is what we observe. We therefore use averaged values for $g_{\mathrm{WW}}^{(2)}$ and $g_{\mathrm{RR}}^{(2)}$ together with the fit results of $g_{\mathrm{WR}}^{(2)}\left(\tau_{\mathrm{D}}\right)$ to plot the above expression for $\mathcal{R}\left(\tau_{\mathrm{D}}\right)$. From $\mathcal{R}$ we define the memory time as the time beyond which write and read light fields are no longer non-classically correlated,

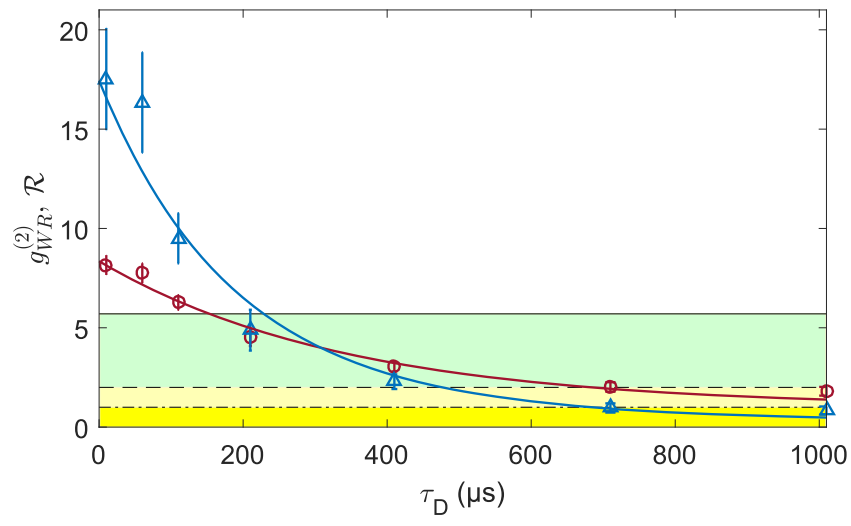

Fig. 4 Photon correlations for delayed readout. Shown are $g_{W R}^{(2)}$ (red) and the Cauchy-Schwarz parameter $\mathcal{R}$ (blue) versus various read pulse delays $\tau_{\mathrm{D}}$ for an integrated read pulse duration of $40 \mu \mathrm{s}$ together with the fit to $g_{\mathrm{WR}}^{(2)}$ (red line) and the resulting $\mathcal{R}$ (blue line). The black line marks the Bellinequality limit $g_{W R}^{(2)} \geq 5.7$ and the dashed line marks the typical nonclassicallity signature $g_{W R}^{(2)}>2$. The dash-dotted line is the formal nonclassicallity criterion $\mathcal{R}>1$. Error bars represent one standard deviation. Source data are provided as a Source Data file.

i.e., not fulfilling $\mathcal{R}>1$ (Fig. 4, dash-dotted line). We use $\mathcal{R}$ as a formal non-classicality bound instead of the typical signature $g_{\mathrm{WR}}^{(2)}>2^{21}$. The corresponding memory time is $\tau_{\mathrm{NC}}^{\mathcal{R}}=(0.68 \pm 0.08) \mathrm{ms}$. The limit for violating the Bell inequality is given by $g_{\mathrm{WR}}^{(2)} \geq 5.7^{43}$. From the fit in Fig. 4 this holds for $\tau_{\mathrm{BI}}=$ $(0.15 \pm 0.03) \mathrm{ms}$.

The non-classical memory time of the atomic ensemble is limited by noise from the atomic decay. However, the retrieval efficiency $\eta_{\mathrm{R}}$ is noise free due to its definition (see Supplementary Note 4), which allows us to determine the $1 / e$-lifetime of the collective excitation in the memory, amounting to $\tau_{\eta_{\mathrm{R}}}=0.89_{-0.23}^{+0.49} \mathrm{~ms}$. The collective excitation lifetime is expected to be limited to half of the transverse macroscopic spin amplitude decay time, separately measured to be $T_{2}=2 \mathrm{~ms}$ (see "Methods" section) and to be dominated by spin relaxation due to wall collisions. On this time scale the atoms experience thousands of wall collisions. Hence, the long lifetime of the targeted symmetric atomic mode demonstrates the effect of the spin-protecting coating.

\section{Discussion}

Our results demonstrate the capability to herald, store and read out a single long-lived collective atomic excitation from a roomtemperature atomic vapor. We verify the single-photon nature of the retrieved light from observing strong photon antibunching. High cross-correlation and near-millisecond storage time at room temperature enable applications in quantum networks, where the platform can be immediately used as a building block for entanglement generation over up to $200 \mathrm{~km}$. The feasibility of this was demonstrated in a recent proof-of-principle study verifying short-range entanglement of two warm atomic vapors after submicrosecond storage through the DLCZ protocol ${ }^{44}$. The technological simplicity of our system facilitates the setup of a multitude of identical parallel systems, and thus a quantum repeater as well as simulator applications. Increasing the cell transverse dimension from the current $300 \mu \mathrm{m}$ to several millimeters and using state-of-the-art coating ${ }^{32}$, we can expect to increase the storage time of single excitations to at least hundreds of milliseconds. The required higher rejection of the excitation light has been demonstrated in our laboratory ${ }^{45}$. Implementing a top-hat optical mode for improved filling of the cell with light ${ }^{46}$ will relax 
the spectral filtering requirements for motional averaging for a larger cell.

Besides the application as a source of narrowband single photons one can explore a larger phase space by accumulating excitations. Exciting applications may also arise from interfacing such quantum-state engineering with other platforms, such as cold atoms or mechanical oscillators.

\section{Methods}

Light. Excitation light pulses for write and read are derived from a narrowband home-built external cavity diode laser at $895 \mathrm{~nm}$. It is locked via a beat-note lock with fixed detuning to the $F=4 \rightarrow F^{\prime}=4$ transition of the $D_{1}$ line of cesium. The write and read locking and excitation frequencies are derived using two AOMs.

Vapor cell. In our experiments we use a cesium vapor cell with an interaction volume of $300 \mu \mathrm{m} \times 300 \mu \mathrm{m} \times 10 \mathrm{~mm}$, coated with a spin-preserving anti-relaxation coating (alkane). The cell cavity mode has a $90 \mu \mathrm{m}$ waist radius $\left(1 / e^{2}\right.$ intensity) at the cell center. Using magneto-optical resonance spectroscopy ${ }^{47}$, the coherence time of the ground-state Zeeman levels was determined to be $T_{2}=2 \mathrm{~ms}$ for an operational temperature of $43^{\circ} \mathrm{C}$ of the experiment and a Zeeman splitting of $v_{\mathrm{L}}=$ $2.4 \mathrm{MHz}$.

Data acquisition. To compensate for drifts in the experimental setup while acquiring measurement data, sequences with and without write, as well as sequences with varying delay $\tau_{\mathrm{D}}$ are interleaved. Sequences without preceding write pulse are used to estimate noise levels in the readout.

Optical pumping. During the locking and optical pumping window in the experimental sequence, the coherent atomic spin state is prepared using two circularly polarized pump and repump beams. The pumping is parallel to the magnetic field orientation. The repump laser is locked onto the $F=3 \rightarrow F^{\prime}=2,3$ crossover transition, while the pump laser is locked on the transition $F=4 \rightarrow F^{\prime}=4$. We determine the atomic polarization of atoms in the $F=4$ manifold (typically $>99.2 \%$ ) using pulsed magneto-optical resonance spectroscopy. This high polarization is achieved by optimized beam geometry and by turning off the repump laser first, and keeping the pump laser turned on for a few microseconds longer.

Polarization and spectral filtering. The leakage contribution is minimized using a half wave plate and a quarter wave plate after the cell cavity to optimize the polarization orientation, such that the polarization filtering using a GlanThompson polarizer reaches a suppression of $5 \times 10^{-5}$. Following this polarization filtering stage, the spectral filtering consisting of two cavities for each of the detection setups, provides around $60 \mathrm{~dB}$ suppression for both detection setups.

Magic detuning. FWM noise is due to read excitation light coupling to the state $|4,4\rangle$ and via a spontaneous Raman process creating excess excitations in $|4,3\rangle$. The associated Raman-Rabi coupling is given by $R \propto g \Omega / \Delta$, where $\Delta$ is the detuning, and $\Omega(g)$ is the coupling strength for the excitation field (scattered field), respectively. These coupling strengths include the Clebsch-Gordan coefficients for the corresponding transitions. For the Raman transition coupled to multiple excited states $m$ we need to sum over their contributions to the coupling $R \propto \sum_{\mathrm{m}} g_{\mathrm{m}} \Omega_{\mathrm{m}} / \Delta_{\mathrm{m}}$, where $\Delta_{\mathrm{m}}$ is the detuning from the respective state. For Raman transitions with Clebsch-Gordan coefficients of opposite signs, there will be a detuning where the above sum vanishes. For cesium atoms and light on the $D_{1}$ line with a Raman transition between the states $|4,4\rangle$ and $|4,3\rangle$ via the excited states $\left|4^{\prime}, 3\right\rangle$ and $\left|3^{\prime}, 3\right\rangle$, the detuning where this transition is effectively suppressed lies outside the Dopplerbroadened width. Including the motion of the atoms we can follow the derivation for motional averaging from ${ }^{31}$, adding the relevant excited states. This yields the expression for the coupling $R \propto \sum_{\mathrm{m}} g_{\mathrm{m}} \Omega_{\mathrm{m}} w\left[\left(\Delta_{\mathrm{m}}+i \gamma / 2\right) / \Gamma_{\mathrm{D}}\right]$ with the Faddeeva function $w[z]$, the natural linewidth $\gamma$ and the Doppler broadening $\Gamma_{\mathrm{D}}$. We find an optimal FWM suppression at a detuning of $\Delta_{4^{\prime}}=924 \mathrm{MHz}$.

Intrinsic retrieval efficiency. From the correlation model (see Supplementary Note 3$)$ we find the fit parameter $\eta_{\mathrm{Y}}=(6.0 \pm 0.2) \%$, which is the probability to have a detection event caused by retrieving one collective excitation in the symmetric atomic mode. Thus, it includes propagation losses, i.e., $\eta_{Y}=\eta_{\mathrm{d}} \eta_{\mathrm{esc}} \eta_{\mathrm{R}}^{*}$. To estimate the intrinsic retrieval efficiency $\eta_{\mathrm{R}}^{*}$ we correct for the losses from two parts of the setup: 1) The efficiency $\eta_{\mathrm{esc}}$ of a photon generated inside the cell cavity escaping out through the outcoupling mirror. It is found from the single-pass transmission through the vapor cell $T_{\text {cell }}$ and the reflectivity $R$ of the outcoupling mirror as $\eta_{\text {esc }} \approx T_{\text {cell }}(1-R) /\left(1-R T_{\text {cell }}^{2}\right)=(45 \pm 2) \%$. 2) The detection efficiency of light in the retrieval photon mode after the cell cavity. We determine this using strongly attenuated laser light in the retrieval photon mode to be $\eta_{\mathrm{d}}=(19 \pm 2) \%$.
Uncertainty estimation. The error bars for conditional auto-correlation functions and the cross-correlation functions, as well as the retrieval efficiency are calculated using Poissonian errors. An exception is the error bar on the $g_{\mathrm{RR} \mid \mathrm{W}=1}^{(2)}=0$ point in Fig. $3 \mathrm{c}$ for the conditional auto-correlation, where no conditional double read detection event was recorded. In this case, we assign an error bar of $g_{\mathrm{RR} \mid \mathrm{W}=1}^{(2)}$ equal to the value if one conditional double read detection event had been recorded.

\section{Data availability}

The data that support the findings of this study are available from the corresponding author upon reasonable request. Figure Source data are provided with this paper.

Received: 14 April 2021; Accepted: 28 May 2021;

Published online: 17 June 2021

\section{References}

1. Aharonovich, I., Englund, D. \& Toth, M. Solid-state single-photon emitters. Nat. Photonics 10, 631-641 (2016).

2. Awschalom, D. D., Hanson, R., Wrachtrup, J. \& Zhou, B. B. Quantum technologies with optically interfaced solid-state spins. Nat. Photonics 12, 516-527 (2018).

3. Wan, N. H. et al. Large-scale integration of artificial atoms in hybrid photonic circuits. Nature 583, 226-231 (2020).

4. Ding, X. et al. On-demand single photons with high extraction efficiency and near-unity indistinguishability from a resonantly driven quantum dot in a micropillar. Phys. Rev. Lett. 116, 020401 (2016).

5. Kiršanskè, G. et al. Indistinguishable and efficient single photons from a quantum dot in a planar nanobeam waveguide. Phys. Rev. B 96, 165306 (2017).

6. Laplane, C., Jobez, P., Etesse, J., Gisin, N. \& Afzelius, M. Multimode and longlived quantum correlations between photons and spins in a crystal. Phys. Rev. Lett. 118, 210501 (2017).

7. Kutluer, K., Mazzera, M. \& De Riedmatten, H. Solid-state source of nonclassical photon pairs with embedded multimode quantum memory. Phys Rev. Lett. 118, 210502 (2017).

8. Lukishova, S. G. \& Bissell, L. J. Nanophotonic Advances for Room-Temperature Single-Photon Sources (pp. 103-178. Springer, 2019).

9. Mücke, M. et al. Generation of single photons from an atom-cavity system. Phys. Rev. A 87, 063805 (2013).

10. Radnaev, A. G. et al. A quantum memory with telecom-wavelength conversion. Nat. Phys. 6, 894-899 (2010).

11. Corzo, N. V. et al. Waveguide-coupled single collective excitation of atomic arrays. Nature 566, 359-362 (2019).

12. Yang, S. J., Wang, X. J., Bao, X. H. \& Pan, J. W. An efficient quantum lightmatter interface with sub-second lifetime. Nat. Photonics 10, 381-384 (2016)

13. Bimbard, E. et al. Homodyne tomography of a single photon retrieved on demand from a cavity-enhanced cold atom memory. Phys. Rev. Lett. 112, 033601 (2014).

14. Shu, C. et al. Subnatural-linewidth biphotons from a Doppler-broadened hot atomic vapour cell. Nat. Commun. 7, 12783 (2016).

15. Eisaman, M. D. et al. Electromagnetically induced transparency with tunable single-photon pulses. Nature 438, 837-841 (2005).

16. Ripka, F., Kübler, H., Löw, R. \& Pfau, T. A room-temperature single-photon source based on strongly interacting Rydberg atoms. Science 362, 446-449 (2018).

17. Finkelstein, R., Poem, E., Michel, O., Lahad, O. \& Firstenberg, O. Fast, noisefree memory for photon synchronization at room temperature. Sci. Adv. 4, eaap8598 (2018).

18. Kaczmarek, K. T. et al. High-speed noise-free optical quantum memory. Phys. Rev. A 97, 042316 (2018).

19. Duan, L. M., Lukin, M. D., Cirac, J. I. \& Zoller, P. Long-distance quantum communication with atomic ensembles and linear optics. Nature 414, 413-418 (2001).

20. Nunn, J. et al. Enhancing multiphoton rates with quantum memories. Phys. Rev. Lett. 110, 133601 (2013).

21. Sangouard, N., Simon, C., De Riedmatten, H. \& Gisin, N. Quantum repeater based on atomic ensembles and linear optics. Rev. Modern Phys. 83, 33-80 (2011).

22. Bashkansky, M., Fatemi, F. K. \& Vurgaftman, I. Quantum memory in warm rubidium vapor with buffer gas. Opt. Lett. 37, 142-144 (2012).

23. Dou, J. P. et al. A broadband DLCZ quantum memory in room-temperature atoms. Commun. Phys. 1, 55 (2018)

24. Pang, X. L. et al. A hybrid quantum memory-enabled network at room temperature. Sci. Adv. 6, eaax1425 (2020).

25. Zugenmaier, M., Dideriksen, K. B., Sørensen, A. S., Albrecht, B. \& Polzik, E. S. Long-lived non-classical correlations towards quantum communication at room temperature. Commun. Phys. 1, 76 (2018). 
26. Namazi, M., Kupchak, C., Jordaan, B., Shahrokhshahi, R. \& Figueroa, E. Ultralow-noise room-temperature quantum memory for polarization qubits. Phys. Rev. Appl. 8, 034023 (2017).

27. Reim, K. F. et al. Single-photon-level quantum memory at room temperature. Phys. Rev. Lett. 107, 053603 (2011).

28. Hosseini, M., Campbell, G., Sparkes, B. M., Lam, P. K. \& Buchler, B. C. Unconditional room-temperature quantum memory. Nat. Phys. 7, 794-798 (2011).

29. Julsgaard, B., Sherson, J., Cirac, J. I., Fiurášek, J. \& Polzik, E. S. Experimental demonstration of quantum memory for light. Nature 432, 482-486 (2004).

30. Katz, O. \& Firstenberg, O. Light storage for one second in room-temperature alkali vapor. Nat. Commun. 9, 2074 (2018).

31. Borregaard, J. et al. Scalable photonic network architecture based on motional averaging in room temperature gas. Nat. Commun. 7, 11356 (2016).

32. Balabas, M. V., Karaulanov, T., Ledbetter, M. P. \& Budker, D. Polarized alkalimetal vapor with minute-long transverse spin-relaxation time. Phys. Rev. Lett. 105, 070801 (2010).

33. Dicke, R. H. Coherence in spontaneous radiation processes. Phys. Rev. 93, 99-110 (1954).

34. Michelberger, P. S. et al. Interfacing GHz-bandwidth heralded single photons with a warm vapour Raman memory. New J. Phys. 17, 043006 (2015).

35. Walther, P. et al. Generation of narrow-bandwidth single photons using electromagnetically induced transparency in atomic ensembles. Int. J. Quantum Inf. 5, 51-62 (2007).

36. Saunders, D. J. et al. Cavity-enhanced room-temperature broadband raman memory. Phys. Rev. Lett. 116, 090501 (2016).

37. Thomas, S. E. et al. Raman quantum memory with built-in suppression of four-wave-mixing noise. Phys. Rev. A 100, 33801 (2019).

38. Romanov, G., O’Brien, C. \& Novikova, I. Suppression of the four-wave mixing amplification via Raman absorption. J. Mod. Opt. 63, 2048-2057 (2016).

39. Zhang, K. et al. Suppression of the four-wave-mixing background noise in a quantum memory retrieval process by channel blocking. Phys. Rev. A 90 , 033823 (2014).

40. Vurgaftman, I. \& Bashkansky, M. Suppressing four-wave mixing in warmatomic-vapor quantum memory. Phys. Rev. A 87, 063836 (2013).

41. Dạbrowski, M., Chrapkiewicz, R. \& Wasilewski, W. Hamiltonian design in readout from room-temperature Raman atomic memory. Opt. Express 22 26076 (2014)

42. Clauser, J. F. Experimental distinction between the quantum and classical field-theoretic predictions for the photoelectric effect. Phys. Rev. D 9, 853-860 (1974).

43. Wallucks, A., Marinković, I., Hensen, B., Stockill, R. \& Gröblacher, S. A quantum memory at telecom wavelengths. Nat. Phys. 16, 772-777 (2020).

44. $\mathrm{Li}, \mathrm{H}$. et al. Heralding quantum entanglement between two room-temperature atomic ensembles. Preprint at https://arxiv.org/abs/2007.10948 (2020).

45. Galinskiy, I., Tsaturyan, Y., Parniak, M. \& Polzik, E. S. Phonon counting thermometry of an ultracoherent membrane resonator near its motional ground state. Optica 7, 718-725 (2020).

46. Jensen, K. et al. Quantum memory for entangled continuous-variable states. Nat. Phys. 7, 13-16 (2011).
47. Julsgaard, B., Sherson, J., Sørensen, J. L. \& Polzik, E. S. Characterizing the spin state of an atomic ensemble using the magneto-optical resonance method. J. Opt. B 6, 5-14 (2004).

\section{Acknowledgements}

The authors acknowledge helpful discussions with Michal Parniak and support with the design of cavities by Ivan Galinskiy. The alkane coated cell has been fabricated by Mikhail Balabas. This project has been supported by the European Research Council Advanced grant QUANTUM-N, the Villum Foundation, and John Templeton Foundation.

\section{Author contributions}

E.S.P. conceived and led the project. K.B.D., R.S., and M.Z. have contributed equally to the work, built the experiment, took the data and performed the analysis. All authors discussed the results and participated in the writing process of this manuscript.

\section{Competing interests}

The authors declare no competing interests.

\section{Additional information}

Supplementary information The online version contains supplementary material available at https://doi.org/10.1038/s41467-021-24033-8.

Correspondence and requests for materials should be addressed to E.S.P.

Peer review information Nature Communications thanks the anonymous reviewer(s) for their contribution to the peer review of this work. Peer reviewer reports are available

Reprints and permission information is available at http://www.nature.com/reprints

Publisher's note Springer Nature remains neutral with regard to jurisdictional claims in published maps and institutional affiliations.

Open Access This article is licensed under a Creative Commons Attribution 4.0 International License, which permits use, sharing, adaptation, distribution and reproduction in any medium or format, as long as you give appropriate credit to the original author(s) and the source, provide a link to the Creative Commons license, and indicate if changes were made. The images or other third party material in this article are included in the article's Creative Commons license, unless indicated otherwise in a credit line to the material. If material is not included in the article's Creative Commons license and your intended use is not permitted by statutory regulation or exceeds the permitted use, you will need to obtain permission directly from the copyright holder. To view a copy of this license, visit http://creativecommons.org/ licenses/by/4.0/

(C) The Author(s) 2021 